\title{
Content based Image Retrievals for Brain Related Diseases
}

\author{
T.V. Madhusudhana Rao \\ Department of CSE, \\ T.P.I.S.T., Bobbili, \\ Andhra Pradesh, INDIA
}

\author{
S. Pallam Setty \\ Department of CS\&SE, \\ Andhra University, \\ Visakhapatnam, INDIA
}

\author{
Y. Srinivas \\ Department of IT, \\ GITAM University, \\ Visakhapatnam, INDIA
}

\begin{abstract}
This paper is attributed to develop a methodology for assisting the radiologists in proper identification of the diseases. In this paper we have considered the brain related disease archive from University of Rennes 1 database to assist in finding the similar relevance images to the radiologists thereby helping them in drawing conclusions in their clinical practices. The methodology is implemented by considering the Generalized Gamma Distribution wherein the features extracted from the images using Discrete Cosine Transformation. KullbackLeibler (KL) divergence methodology is used for finding the most similar images. The query image is processed and relevant images are retrieved. The final estimates of the parameters of Generalized Gamma Distribution are derived and the retrieved accuracy is identified using metrics.
\end{abstract}

\section{Keywords}

Generalized Gamma Distribution, image retrieval, KL divergence, Discrete Cosine Transformation, performance metrics

\section{INTRODUCTION}

Today according to latest statistics, the mortality rate has increased due to brain diseases. Among the various diseases related to the brain, diseases like Brain Tumor, Brain Hemorrhage are focused at large. In order to interpret this disease accurately, MRI scannings are mostly preferred by the doctors. This is due to advantage of the non-ionizing property of MRI. These images are subject to report formation by the radiologist. However, with the immense increase of the cases, a burden has increased on the radiologist since majority of the report utilizing is based on the visual interpretation from the radiologist.

As the cases increases, since the proportionality of the radiologist is lagging there is every possibility of wrong/inaccurate diagnosis due to sensitivity of the human eye. Therefore effective methodologies are to be developed to help the radiologist at the time of diagnosis.

Hence this paper makes an attempt in this direction. A archive of medical images with cross-section of brain diseases is maintained. The patients MRI images are considered as query image and the features are extracted using DCT coefficient presented in Section 2 of the paper. These features are subjected to a model built using Generalized Gamma Distribution. The details about the Generalized Gamma Distribution together with its updated estimates are presented in Section 3 of the paper. In order to minimize the searches $\mathrm{KL}$ divergence algorithm is proposed in Section 4 of the paper. In Section 5 of the paper deals with the methodology and the results derived are presented with evaluation metrics in Section 6. This paper is concluded in Section 7. Scope for further work is presented in Section 8.

\section{DISCRETE COSINE TRANSFORMATION}

The DCT methodology is utilized in this paper for reducing the dimensionality and identifying a feature vector [1], [2], [3], [4], [5]. In order to utilize the DCT, mid level features like considering the texture and shape will be more effective over considering the low level features like color features, coefficients .etc., [6], [7]. The DCT divides image into blocks of $8 \mathrm{X} 8$ and the coefficients are extracted using the formula (1)

$$
\begin{aligned}
& \mathrm{C}(\mathrm{u}, \mathrm{v})= \\
& \frac{1}{4} \mathrm{k}(\mathrm{u}) \mathrm{k}(\mathrm{v}) \sum_{\mathrm{i}=0}^{7} \sum_{\mathrm{j}=0}^{7} \mathrm{x}(\mathrm{i}, \mathrm{j}) \operatorname{Cos}\left(\frac{\left(2_{\mathrm{i}}+1\right) \mathrm{u} \pi}{16}\right) \operatorname{Cos}\left(\frac{\left(2_{\mathrm{j}}+1\right) \mathrm{v} \pi}{16}\right) \\
& \mathrm{k}(\mathrm{u}), \mathrm{K}(\mathrm{v})=\left\{\begin{array}{lc}
1 / 1) \\
\sqrt{2} & \text { if } \mathrm{u} \text { and } \mathrm{v}=0 \\
1 & \text { otherwise } 0
\end{array}\right.
\end{aligned}
$$

Where $0 \leq \mathrm{u}, \mathrm{v} \leq 7$ and $\mathrm{x}(\mathrm{i}, \mathrm{j})$ is the pixel intensity value at location i, j. $\mathrm{C}(0,0)$ is known as low frequency (DC) and the remaining 63 coefficients are known as high frequency coefficients (ACs).

\section{GENERALIZEDGAMMA DISTRIBUTION}

In order to classify the images accurately, the features extracted from Section 2 are given as input to Generalized Gamma Distribution presented in equation (2).

$f(x, k, c, a, b)=\frac{c(x-a)^{c k-1} e^{-\left(\frac{x-a}{b}\right)^{c}}}{b^{c k} \Gamma(k)}$

Where $\mathrm{a}, \mathrm{b}, \mathrm{c}, \mathrm{k}$ are called the gamma variants and $\mathrm{c}, \mathrm{k}$ are called shape parameters such that $\mathrm{c}, \mathrm{k}>0$ and $\mathrm{a}$ is the location partner, and $\mathrm{b}$ is the scale parameter with $\mathrm{a}, \mathrm{b}>0$.

The K-means algorithm is utilized to identify the updated equations for the model parameters are given in equation (3) to (6).

$$
\begin{aligned}
& \mathrm{c}=\frac{1}{\frac{1 \partial \Gamma}{\Gamma \partial \mathrm{c}}-\mathrm{klog}\left(\frac{\mathrm{x}-\mathrm{a}}{\mathrm{b}}\right)+\frac{(\mathrm{x}-\mathrm{a})^{\mathrm{c}}}{\mathrm{b}^{c} \log \left(\frac{\mathrm{x}-\mathrm{a}}{\mathrm{b}}\right)}} \\
& \mathrm{a}=\mathrm{x}-\frac{1-\mathrm{ck}}{\frac{1 \partial \Gamma}{\Gamma \partial \mathrm{a}}-\frac{\mathrm{c}}{\mathrm{b}}\left(\frac{\mathrm{x}-\mathrm{a}}{\mathrm{b}}\right)^{\mathrm{c}-1}} \\
& \mathrm{~b}=\frac{\mathrm{ck}}{\frac{\mathrm{c}}{\mathrm{b}^{c+1}}(\mathrm{x}-\mathrm{a})^{\mathrm{c}}-\frac{1 \partial \Gamma}{\Gamma \partial \mathrm{b}}} \\
& \mathrm{k}=1+\frac{\left[\int_{0}^{\infty} \mathrm{e}^{-\mathrm{t}}\left(\log _{\mathrm{e}} \mathrm{t}\right) \mathrm{t}^{\mathrm{k}-1} \mathrm{dt}\right]}{\Gamma(\mathrm{k}-1)\left[\operatorname{clog}\left(\frac{\mathrm{x}-\mathrm{a}}{\mathrm{b}}\right)-\frac{10 \Gamma}{\Gamma \partial \mathrm{k}}\right]}
\end{aligned}
$$

The detailed derivations of this equation are presented in Appendix-I.

The main advantage of utilizing the Gamma distribution is that the query image/ images with deformity will have high 
intensity values, however, these high intensity pixels will be less in number compared to other pixels there by the distribution is focused with long tails [8]. Hence to interact with these long tails Gamma distribution will be more appropriate [9]. The Gamma distribution consists of several other distributions like Log-Normal, Chi-square, Weibull, Exponential distributions as particular cases. Another due advantage of utilizing the Gamma distribution is its ability to identify the medical image feature in presence of noise.

\section{RELEVANT IMAGE IDENTIFICATION USING KL DIVERGENCE METHOD}

The proposed paper is to facilitate the radiologist to diagnose the disease more accurately with minimum time stamp. In order to achieve this, the search space should be minimized. Many algorithms are projected in this direction. Among which KL divergence [10] is utilized in this paper. The KL divergence algorithm is given by equation (7).
$\operatorname{KL}\left(\mathrm{p}_{1}, \mathrm{p}_{2}\right)=\int \mathrm{p}_{1}(\mathrm{x}) \log \left(\frac{\mathrm{p}_{1}(\mathrm{x})}{\mathrm{p}_{2}(\mathrm{x})}\right) \mathrm{dx}$

Where ' $p 1$ ', ' $\mathrm{p} 2$ ' are the two Probability Density Functions computed on different brain images, formulated using Generalized Gamma Distribution.

\section{METHODOLOGY}

The proposed methodology is used to assist the doctors in identifying the diseases more accurately. In this process the archive of diseased medical images shown in Figure 1 are maintained.

In order to minimize the search time the query image is considered and the feature vector is identified after processing the DCT presented in Section 2 of the paper. The PDFs of the images are calculated using the Generalized Gamma Distribution proposed in Section 3. The relevant images are identified using Section 4 and the retrieved images are obtained and are presented in Figure 2.

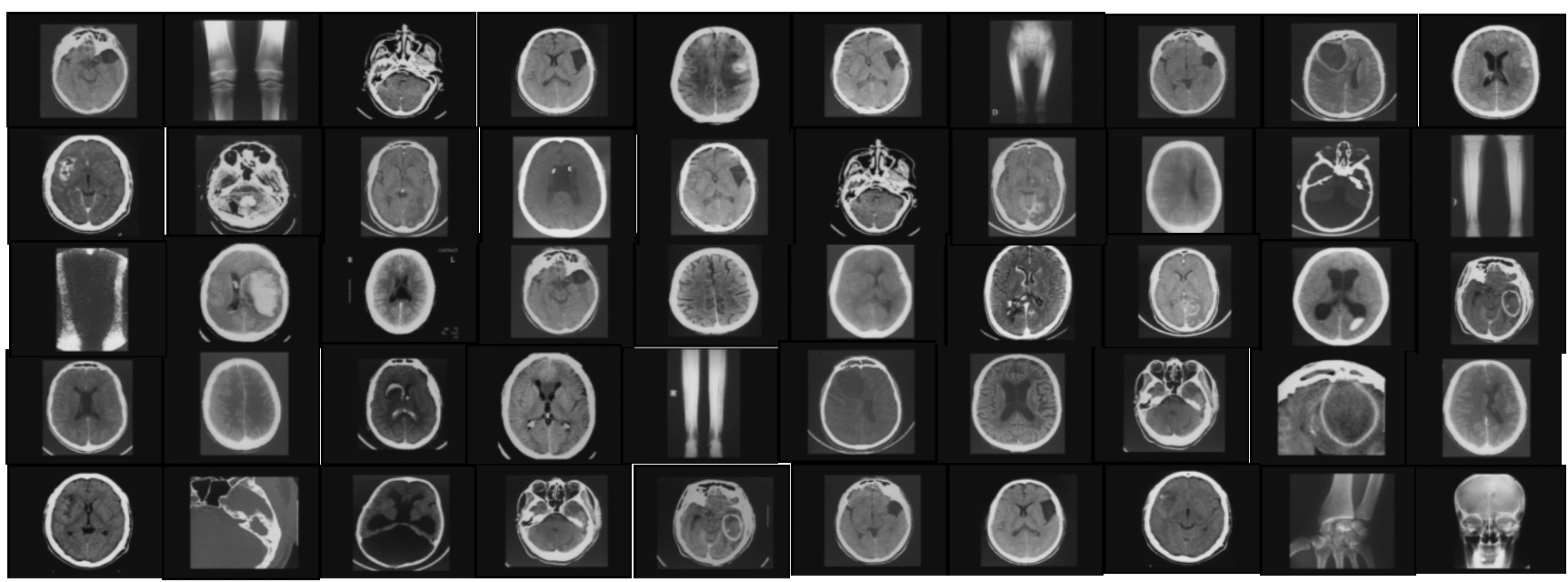

Fig 1: Image Dataset

\begin{tabular}{|c|c|c|c|}
\hline Disease & $\begin{array}{l}\text { Query } \\
\text { image }\end{array}$ & Relevant images & Non-Relevant images \\
\hline Brain tumor & & & \\
\hline $\begin{array}{c}\text { Brain } \\
\text { Hemorrhage }\end{array}$ & & & \\
\hline
\end{tabular}

Fig 2: Input Query Image versus Retrieved Relevant and Non-Relevant images

\section{PERFORMANCE EVALUATION}

The outputs derived against the query processed are evaluated using metrics like Precision and Recall. Precision is the ratio of the number of relevant images retrieved to the total number of irrelevant and relevant images retrieved. It is usually expressed as a percentage.

$$
\text { PRECISION }=(\mathrm{A} / \mathrm{A}+\mathrm{C}) * 100
$$

A: Number of relevant images retrieved.

C: Number of irrelevant images retrieved.
A + C: Total number of irrelevant + relevant images retrieved Whereas, Recall is the ratio of the number of relevant images retrieved to the total number of relevant images in the database. It is usually expressed as a percentage.

RECALL $=(\mathrm{A} / \mathrm{A}+\mathrm{B}) * 100$

A: Number of relevant images retrieved B: Number of relevant images not retrieved $\mathrm{A}+\mathrm{B}$ : The total number of relevant images 
The outputs derived using the proposed metrics are presented in Table 1 and in Figure 3 and Figure 4.

Table 1. Precision and Recall based on relevant and nonrelevant images

\begin{tabular}{|c|c|c|c|c|}
\hline $\begin{array}{l}\text { Number of } \\
\text { relevant } \\
\begin{array}{l}\text { images in } \\
\text { database }\end{array}\end{array}$ & $\begin{array}{l}\text { Number of } \\
\text { non- } \\
\text { relevant } \\
\text { images in } \\
\text { database }\end{array}$ & $\begin{array}{l}\text { Ratio of } \\
\text { relevance } \\
\text { to non- } \\
\text { relevance }\end{array}$ & Precision & Recall \\
\hline 8 & 50 & 0.16 & 80 & 6 \\
7 & 50 & 0.14 & 74 & 26 \\
6 & 50 & 0.12 & 62 & 35 \\
5 & 50 & 0.1 & 53 & 42 \\
4 & 50 & 0.08 & 42 & 53 \\
3 & 50 & 0.06 & 35 & 62 \\
2 & 50 & 0.04 & 26 & 74 \\
1 & 50 & 0.02 & 6 & 80 \\
\hline
\end{tabular}

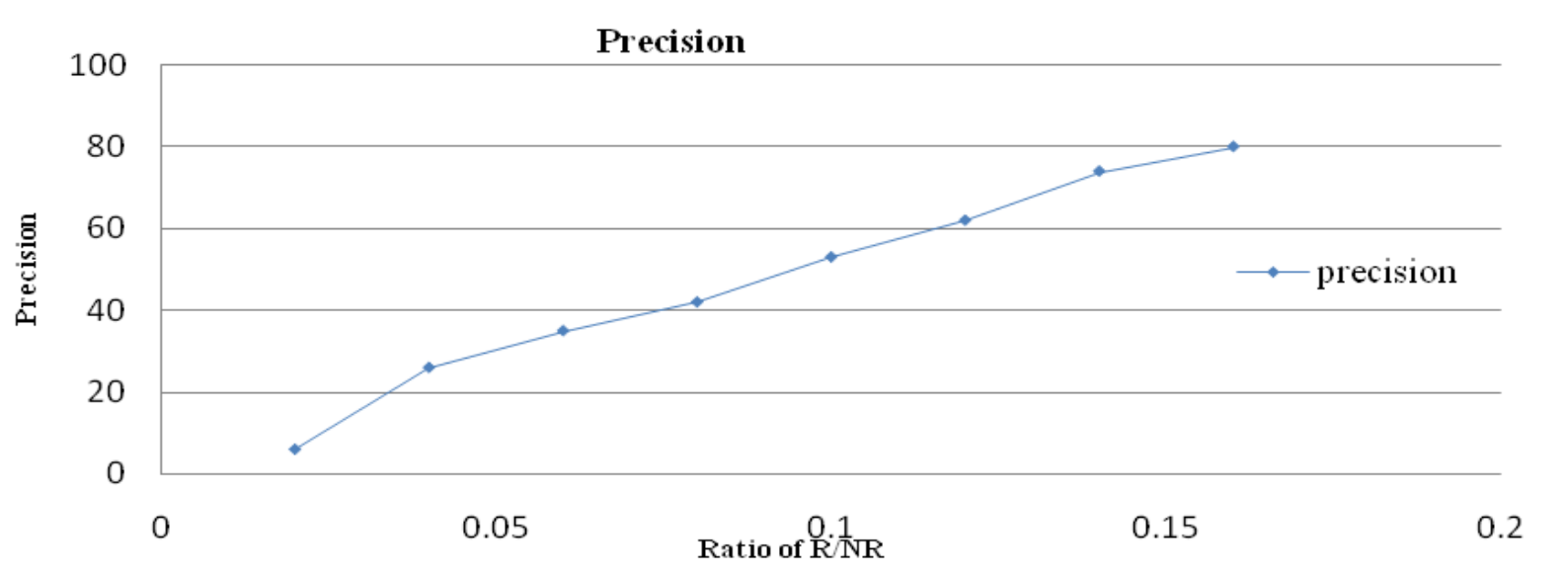

Fig 3: Variation of Precision value with relevant to non-relevant images ratio

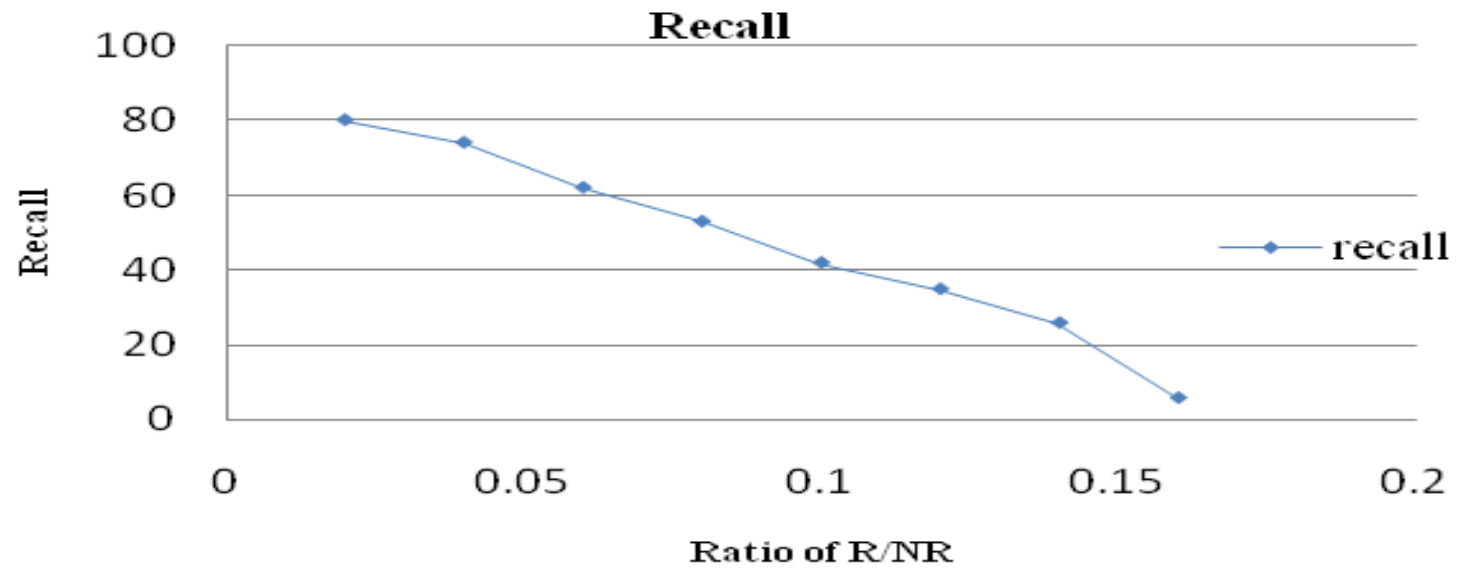

Fig 4: Variation of Recall value with relevant to non-relevant images ratio 
From the above Figure 3 and Figure 4, it can be easily understood that the developed model performs much better on large data sets.

From the developed methodology it can be easily seen that, the developed method performs well and its accuracy rate is increased if the number of trained images are more.

\section{CONCLUSION}

A methodology of retrieving the most relevant images is proposed using DCT coefficients and Generalized Gamma Distribution. This methodology helps to identify the relevant images by compressing the data wherein the repeated informat6ion can be eliminated. This reduces the search time as well as storage time. The database so formulated is checked for relevancy basing on the query image. The relevant images are extracted thereby minimizing the search time. This methodology will be very much useful for radiologists and patients in remote areas where super specialized facility lack. The performance of the model is evaluated using Precision and Recall and developed model exhibits good results.

\section{SCOPE FOR FURTHER RESEARCH}

This work is projected towards the identification of brain diseases based on the content using DCT technology. Since DCT is a lossy compression, effective techniques of addressing these methodologies are to be identified. Usage of wavelet transformation is an alternative which needs further analysis.

\section{REFERENCES}

[1] Nezamabadi-Pour, H. and Saryazdi, S. 2005. Object based image indexing and retrieval in DCT domain using clustering techniques. In Proceedings of World Academy of Science Engineering and Technology.

[2] Veltkamp, R. C. and Tanase, M. 2002. Content-based image retrieval systems: A survey. Department of Computing Science, Utrecht University, 1-62.

[3] Wang, J. andJi, L. 2001. A region and data hiding based error concealment scheme for images. IEEE Transactions on Consumer Electronics, 47(2), 257-262.

[4] Bajaj, M. and Lay, J. A. 2007. Image Indexing and Retrieval in Compressed Domain Using Color Clusters.IEEE Symposium on Computational Intelligence in Image and Signal Processing, CIISP 2007. pp. 271-274.

[5] Smeulders, A. W., Worring, M., Santini, S., Gupta, A.and Jain, R. 2000. Content-based image retrieval at the end of the early years. IEEE Transactions on Pattern Analysis and Machine Intelligence, 22(12), 1349-1380.

[6] Al-Jubouri, H., Du, H. and Sellahewa, H. 2012. Applying Gaussian mixture model on Discrete Cosine features for image segmentation and classification.4thIEEEConferenceon Computer Science and Electronic Engineering (CEEC), pp. 194-199.

[7] Al-Jubouri, H., Du, H. and Sellahewa, H. 2013. Adaptive clustering based segmentation for image classification. 5th IEEE Conference on Computer Science and Electronic Engineering (CEEC) pp. 128-133.

[8] deVes, E., Benavent, X., Ruedin, A., Acevedo, D. and Seijas, L. 2010. Wavelet-based Texture Retrieval Modeling the Magnitudes of Wavelet Detail Coefficients with a Generalized Gamma
Distribution.20thIEEEInternational on Pattern Recognition (ICPR), pp. 221-224.

[9] Kai-Sheng, S. 2008. Globally Convergent Algorithms for Estimating Generalized Gamma Distributions in Fast Signal and Image Processing, IEEE Transactions on Image Processing, 17 (8) 1233-1250.

[10] Goldberger, J., Gordon, S. and Greenspan, H. 2003. An efficient image similarity measure based on approximations of KL-divergence between two gaussian mixtures. InProceedings of theNinth IEEE International Conference on Computer Vision,vol.1, pp. 487-493.

\section{APPENDIX - I \\ ESTIMATION OF THE MODEL PARAMETER THROUGH EXPECTATION MAXIMIZATION ALGORITHM}

For effective retrieval of the relevant images, the parameters of the medical images are to be estimated effectively. For estimating the parameters Expectation Maximization algorithm is used. It maximizes the likelihood of the model.

Let $x_{i}=x_{1}, x_{2}, \ldots, x_{n}$ be the features of the image to be retrieved and which are characterized by using the Generalized Gamma Distribution given in equation (1).

$f(x, k, c, a, b)=\frac{c(x-a)^{c k-1} e^{-\left(\frac{x-a}{b}\right)^{c}}}{b^{c k} \Gamma(k)}$

The likelihood of relevant images is drawn from the set of images. $\mathrm{x}_{1}, \mathrm{x}_{2} \ldots \mathrm{x}_{\mathrm{n}}$ with probability function

$\mathrm{h}(\mathrm{Z}, \theta)=\sum_{\mathrm{i}=1}^{\mathrm{k}} \alpha_{\mathrm{i}} \mathrm{g}_{\mathrm{i}}\left(\mathrm{Z}_{\mathrm{s}}, \theta\right)$

is given by

$$
\begin{aligned}
\mathrm{L}(\theta)= & \prod_{\mathrm{s}=1}^{\mathrm{N}}\left(\sum_{\mathrm{i}=1}^{\mathrm{k}} \alpha_{\mathrm{i}} \mathrm{g}_{\mathrm{i}}\left(\mathrm{Z}_{\mathrm{s}}, \theta\right)\right) \\
& =\prod_{\mathrm{s}=1}^{\mathrm{N}}\left(\sum_{\mathrm{i}=1}^{\mathrm{k}} \alpha_{\mathrm{i}}\left(\frac{\mathrm{c}(\mathrm{x}-\mathrm{a})^{\mathrm{ck}-1} \mathrm{e}^{-\left(\frac{\mathrm{x}-\mathrm{a}}{\mathrm{b}}\right)^{\mathrm{c}}}}{\mathrm{b}^{\mathrm{ck}} \Gamma(\mathrm{k})}\right)\right)
\end{aligned}
$$

This implies

$$
\begin{aligned}
& =\log \left(\sum_{\mathrm{i}=1}^{\mathrm{k}} \alpha_{\mathrm{i}} \mathrm{g}_{\mathrm{i}}\left(\mathrm{Z}_{\mathrm{s}}, \theta\right)\right) \\
& =\sum_{\mathrm{s}=1}^{\mathrm{N}} \log \left(\sum_{\mathrm{i}=1}^{\mathrm{k}} \alpha_{\mathrm{i}} \mathrm{g}_{\mathrm{i}}\left(\mathrm{Z}_{\mathrm{s}}, \theta\right)\right)
\end{aligned}
$$

In order to solve the EM Algorithms, the first step, requires some reasonable initial estimates and this step is called the Estep and second step iteratively calculates the maximum likelihood estimates of the unknown parameters, $\theta$ and this step is called the M-Step.

E-Step: The Expectation (E) Step can be obtained by considering $\log \mathrm{L}(\theta)$ with respect to the parameter vector $\theta^{(0)}$ is calculated from the observed data ' $Z$ ' as

$$
\begin{aligned}
\mathrm{Q}\left(\theta ; \theta^{(0)}\right) & =\mathrm{E}_{\theta^{(0)}}\{\log \mathrm{L}(\theta) \mid \overline{\mathrm{Z}}\} \\
& =\left(\left(\int_{\mathrm{Z}} \log \mathrm{L}(\theta) \mid \overline{\mathrm{Z}}\right) \mathrm{h}\left(\mathrm{Z}_{\mathrm{s}} ; \theta^{(0)}\right)\right) \mathrm{dz} \\
& =\sum_{\mathrm{s}=1}^{\mathrm{N}} \log \left(\mathrm{Z}_{\mathrm{s}} ; \theta\right) \int_{\mathrm{Z}} \mathrm{h}\left(\mathrm{Z}_{\mathrm{s}} ; \theta^{(0)}\right) \mathrm{dz}
\end{aligned}
$$




$$
=\sum_{s=1}^{\mathrm{N}} \log \left(\sum_{\mathrm{i}=1}^{\mathrm{k}} \alpha_{\mathrm{i}} \mathrm{g}_{\mathrm{i}}\left(\mathrm{Z}_{\mathrm{s}} ; \theta\right)\right)
$$

Given the initial parameter one can compute the density of any observation $\mathrm{Z}_{\mathrm{s}}$ as,

$$
\begin{aligned}
\mathrm{h}\left(\mathrm{Z}_{\mathrm{s}}, \theta\right) & =\sum_{\mathrm{i}=1}^{\mathrm{k}} \prod_{\mathrm{i}}^{(\mathrm{l})} \mathrm{g}_{\mathrm{i}}\left(\mathrm{Z}_{\mathrm{s}}, \theta^{(\mathrm{l})}\right) \\
& =\prod_{\mathrm{s}=1}^{\mathrm{N}}\left(\sum_{\mathrm{i}=1}^{\mathrm{k}} \alpha_{\mathrm{i}}\left(\frac{\mathrm{c}(\mathrm{x}-\mathrm{a})^{\mathrm{ck}-1} \mathrm{e}^{-\left(\frac{\mathrm{x}-\mathrm{a}}{\mathrm{b}}\right)^{\mathrm{c}}}}{\mathrm{b}^{\mathrm{ck}} \Gamma(\mathrm{k})}\right)\right)
\end{aligned}
$$

The conditional probability of feature $Z_{s}$ belonging to a particular image is given by

$$
\begin{aligned}
\mathrm{t}_{\mathrm{k}}\left(\mathrm{Z}_{\mathrm{s}} ; \theta^{(\mathrm{l})}\right) & =\frac{\alpha_{\mathrm{k}}^{(\mathrm{l})} \mathrm{g}_{\mathrm{i}}\left(\mathrm{z}_{\mathrm{s}}, \theta^{(\mathrm{l})}\right)}{\mathrm{h}\left(\mathrm{z}_{\mathrm{s}}, \theta^{(\mathrm{l})}\right)} \\
= & \frac{\alpha_{\mathrm{k}}^{(1)} \mathrm{g}_{\mathrm{k}}\left(\mathrm{z}_{\mathrm{s}}, \theta^{(\mathrm{l})}\right)}{\sum_{\mathrm{i}=1}^{\mathrm{k}} \alpha_{\mathrm{i}}^{(1)} \mathrm{g}_{\mathrm{i}}\left(\mathrm{z}_{\mathrm{s}}, \theta^{(1)}\right)}
\end{aligned}
$$

To evaluate E-Step we have

$\mathrm{Q}\left(\theta ; \theta^{(\mathrm{l})}\right)=\mathrm{E}_{\theta^{(\mathrm{l})}}\{\log \mathrm{L}(\theta)\}$

$\sum_{i=1}^{\mathrm{k}} \sum_{\mathrm{s}=1}^{\mathrm{N}} \mathrm{E}^{(\mathrm{l})}\left\{\mathrm{t}_{\mathrm{i}}\left(\mathrm{Z}, \theta^{(\mathrm{l})}\right)\left(\log \left(\mathrm{g}_{\mathrm{i}}\left(\mathrm{Z}_{\mathrm{s}} ; \theta\right)\right)+\log \alpha^{\mathrm{i}}\right)\right\} \ldots(16)$

Where $g_{i}(Z ; \theta)$ can be obtained from equation (1) and substituting the equations we have

$\sum_{\mathrm{i}=1}^{\mathrm{k}} \sum_{\mathrm{s}=1}^{\mathrm{N}} \mathrm{E}^{(\mathrm{l})}\left\{\mathrm{t}_{\mathrm{i}}\left(\mathrm{Z}, \theta^{(\mathrm{l})}\right)[\log \mathrm{c}-(\mathrm{ck}-1) \log (\mathrm{n}-\right.$

$\mathrm{a}-\mathrm{x}-\mathrm{abc}-\mathrm{cklog} \mathrm{b}-\log \Gamma \mathrm{k}+\log \alpha \mathrm{i}$

M-Step: Using the method of first order Lagrangian type function we have
$L=E^{l}\left[\log L\left(\theta^{(l)}\right)+\lambda\left(1-\sum_{i=1}^{k} \alpha_{i}^{(l)}\right)\right]$

In order to maximize a log-likelihood, one has to find the derivative of $\mathrm{L}$ with respect to $\alpha_{k}$ and equate to zero i.e.

$\frac{\partial \mathrm{l}}{\partial \alpha_{\mathrm{k}}}=0=\frac{\partial}{\partial \mathrm{x}}\left[\mathrm{E}^{(1)}\left[\log \mathrm{L}\left(\theta^{(\mathrm{l})}\right)+\lambda\left(1-\sum_{\mathrm{l}=1}^{\mathrm{k}} \alpha_{\mathrm{i}}^{(\mathrm{l})}\right)\right]\right]=0$

Simplifying the equation (19) the updated equation of $\alpha$ can be obtained such that

$\alpha_{\mathrm{k}}^{(1+1)}=\frac{1}{\mathrm{~N}} \sum_{\mathrm{s}=1}^{\mathrm{N}} \mathrm{E}^{(\mathrm{l})}\left[\mathrm{t}_{\mathrm{k}}\left(\mathrm{Z}_{\mathrm{s}}, \theta^{(\mathrm{l})}\right)\right]$

In order to identify the updated equation $\mathrm{c}, \mathrm{a}, \mathrm{b}$ and $\mathrm{k}$, one has to differentiate $\mathrm{Q}\left(\theta, \theta^{(\mathrm{l})}\right)$ with respect to $\mathrm{c}, \mathrm{a}, \mathrm{b}$ and $\mathrm{k}$ and equating them to zero. The updated equations are therefore obtained as

$$
c=\frac{1}{\frac{1 \partial \Gamma}{\Gamma \partial c}-k \log \left(\frac{x-a}{b}\right)+\frac{(x-a)^{c}}{b^{c} \log \left(\frac{x-a}{b}\right)}}
$$

$a=x-\frac{1-c k}{\frac{1 \partial \Gamma}{\Gamma \partial a}-\frac{c}{b}\left(\frac{x-a}{b}\right)^{c-1}}$

$$
\mathrm{b}=\frac{\mathrm{ck}}{\frac{\mathrm{c}}{\mathrm{b}^{\mathrm{c}+1}}(\mathrm{x}-\mathrm{a})^{\mathrm{c}}-\frac{1 \partial \Gamma}{\Gamma \partial \mathrm{b}}}
$$

$\mathrm{k}=1+\frac{\left[\int_{0}^{\infty} \mathrm{e}^{-\mathrm{t}}\left(\log _{\mathrm{e}} \mathrm{t}\right) \mathrm{t}^{\mathrm{k}-1} \mathrm{dt}\right]}{\Gamma(\mathrm{k}-1)\left[\operatorname{clog}\left(\frac{\mathrm{x}-\mathrm{a}}{\mathrm{b}}\right)-\frac{1 \partial \Gamma}{\Gamma \partial \mathrm{k}}\right]}$ 